\title{
LA ARMADURA TRIDIMENSIONAL PARA LA FÁBRICA ARMADA*
}

\section{(THE TRIDIMENSIONAL REINFORCEMENT FOR THE REINFORCED MASONRY)}

\section{Oliveira, Investigador}

J. C. Dotreppe, Director de la Investigación

F.N.R.S. Profesor Adjunto, Servicio de Puentes y Estructuras - Universidad de Lieja. BÉLGICA

Fecha de recepción: 28-VII-92

\section{RESUMEN}

Dentro de un ámplio programa de investigación desarrollado en la Universidad de Lieja, sobre los componentes de la fábrica armada, bloques, mortero y armaduras, se expone en este artículo la parte referida a la construcción de obras de fábrica con armaduras espaciales "Murfor RE".

Este revolucionario sistema de armaduras prefabricadas de carácter tridimensional, constituidas por dos alambres longitudinales enlazados entre sí mediante cercos a distancias regulares, tiene la ventaja de permitir armar la fábrica de bloques en dos direcciones, vertical y horizontal, durante el sencillo proceso de ejecución de la albañilería por hiladas.

El compuesto resultante obtenido, comparado con las soluciones ya conocidas de fábrica de bloques de hormigón hueco armado, le supera en prestaciones según resulta de la investigación presentada, siendo competitivo frente al hormigón armado en muchos casos, y pudiendo utilizarse también en fábricas de bloques cerámicos de amplias perforaciones.

\section{SUMMARY}

Within an extensive research program developed at the University of Lieja in the components of the reinforced masonry, blocks, mortar and reinforcements, this article deals with the part which refers to building masonry works with special reinforcements "Murfor RE".

This revolutionary system of prefabricated reinforcements of tridimensional character formed by two longitudinal wires bound together through stirrups at regular distances, has the advantage of allowing the block masonry to be reinforced in two directions, vertical and horizontal, during the simple process of execution of bricklaying in courses.

According to this research, the obtained compound result, compared with the solutions already known of block masonries made of reinforced hollow concrete, surpasses them in properties. It is competitive with the reinforced concrete in many cases and can be also used in masonries made of ceramic blocks with wide perforations.

\section{INTRODUCCIÓN}

En la actualidad, la fábrica armada es un sistema de construcción muy utilizado en el continente americano. Las ventajas que presenta esta técnica de construcción empiezan a afectar ciertas tradiciones de construcción en Europa. Varios países europeos tienen ya reglamentaciones y el Euro-Código n.o 6 prepara reglas unificadas en este sector.
En la post-guerra, los desarrollos de las técnicas de construcción de fábrica armada han interesado sobre todo a los países situados en las regiones sometidas a terremotos. Fue en los años 60 cuando la fábrica armada surgió como una alternativa interesante, desde el punto de vista económico, para los problemas planteados por la construcción de viviendas en zonas fuertemente pobladas.

* Estudio sobre la fábrica armada realizado en la Universidad de Lieja con el sistema de armaduras modulares con cercos. 
Debido a la presión lateral ejercida por el viento sobre los edificios, la fábrica ordinaria se limita a alturas que, normalmente, no rebasan las 3 ó 4 plantas. La utilización de armaduras permitió construir edificios que tienen, actualmente, más de 20 plantas.

La técnica de construcción en fábrica armada, como todas las demás, se encuentra en constante desarrollo. Todavía se tienen que mejorar algunos puntos, sobre todo en lo relativo a los medios que permiten que la fábrica tenga un comportamiento mecánico lo más homogéneo posible. Para ello, este documento presenta una serie de recomendaciones extraidas de un estudio realizado en el Servicio de Puentes y Estructuras de la Universidad de Lieja (1988-1992) bajo la dirección de los Profesores R. Baus y J.C. Dotreppe. Estas recomendaciones se refieren a los materiales de fábrica, la dosificación del microhormigón, los procedimientos de realización de la fábrica y la utilización y el dimensionamiento de un sistema de armaduras modulares con cercos.

Tiene especial relevancia en nuevo sistema ensayado con armaduras prefabricadas tridimensionales que analizamos a continuación.

\section{CONSTRUCCIÓN DE UN MURO DE FÁBRICA ARMADA}

En fábrica armada es muy importante respetar un módulo básico con el fin de concebir una construcción económicamente rentable. Para ello, la longitud y la altura de los muros, así como las dimensiones de los huecos deben ser múltipos de $20 \mathrm{~cm}$.

El muro se eleva sobre una viga de reparto que forma parte del cimiento. Cuando lo requiera un terreno deficiente o solicitaciones importantes, se recurrirá al cálculo.

Seguidamente se colocan las hiladas de bloques, hasta media altura o hasta la altura total de una planta (3). Se utilizan bloques especiales de tipo dintel en la última hilada para que sirva de apoyo a los forjados. Estos bloques se utilizan también a veces a media altura para servir de refuerzo.

Se colocan armaduras verticales y horizontales en los huecos de bloque y armaduras horizontales en las juntas de mortero. Se puede utilizar también un sistema de arma- duras modulares en estribo, que consta de armaduras verticales y horizontales al mismo tiempo, ya que está prefabricada tridimensionalmente. Este sistema, que se presenta en el apartado 5, se probó en la Universidad de Lieja y dio resultados satisfactorios.

Según los ensayos de adherencia bloque-microhormigón realizados en la Universidad de Lieja, la resistencia a la adherencia del microhormigón varía a lo largo de la altura del muro y esta heterogeneidad es tanto más importante cuanto más elevada sea la altura de relleno. Esta influencia resulta tanto más acusada cuanto mayor es la relación e/c.

Por consiguiente se tiene interés en alturas de relleno reducidas al máximo. El sistema modular en estribo tridimensional se presta bien a un relleno de los huecos de los bloques a poca altura, lo cual permite un apisonado más eficaz y confiere al muro un estado de compactación más homogéneo. Permite también un montaje del muro de forma continua. Por consiguiente, desde este punto de vista, resulta más favorable.

\section{DIMENSIONAMIENTO DE LOS MUROS DE FÁBRICA ARMADA SOMETIDOS A LA FLEXIÓN}

\section{Sistema tradicional}

El sistema tradicional en mampostería armada se representa en la figura la, b y c. Lo constituye una armadura longitudinal continua dispuesta en los huecos de los ladrillos (Fig. 1a) o de los bloques (Fig. 1b). Por razón de facilidad de colocación, la armadura ocupa por lo general una posición central, a media distancia de las caras del bloque. Para muros de gran altura, resulta a veces necesario prever solapes de armadura. En el caso de muros de ladrillos o de bloques macizos (Fig. 1c), se dispone por lo general de las armaduras en el espacio situado entre dos paredes, que se rellena entonces con una lechada de mortero.

El estudio experimental realizado en la Universidad de Lieja, que se describirá en el apartado siguiente, no tenía como finalidad específica estudiar el sistema tradicional, sino más bien realizar una comparación entre una nueva concepción, el sistema modular con cercos y el sistema tradicional. 


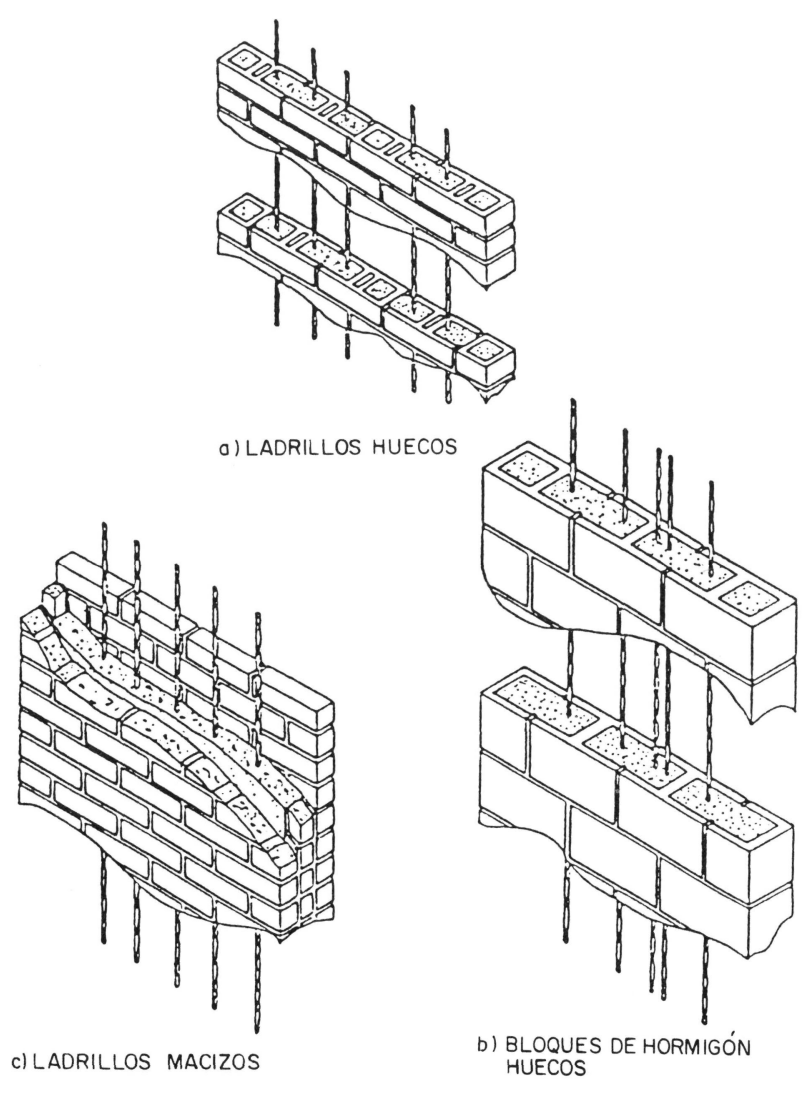

Fig. 1.-Disposición de las armaduras para distintos tipos de fábricas armadas.
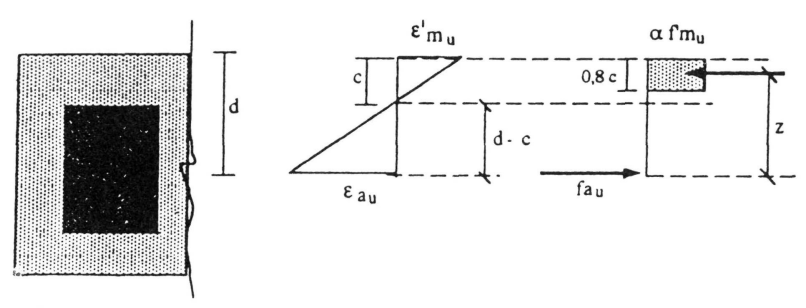

Fig. 2.1.-Distribución de las tensiones y de las deformaciones en un muro armado flexionado. Cálculo en estado límite-último. Sistema tradicional.

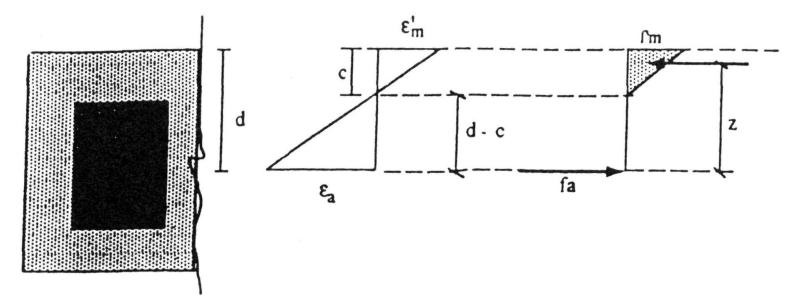

Fig. 2.2.-Distribución de las tensiones y de las deformaciones en un muro flexionado. Método de las tensiones admisibles. Sistema tradicional.
Los resultados obtenidos sobre muros sometidos a flexión transversalmente confirman los esquemas habitualmente utilizados para la evaluación de las características siguientes:

- la resistencia sobre base del método de los estados límites últimos (Fig. 2.1.);

- la resistencia sobre base del método de las tunsiones admisibles (Fig. 2.2);

- las condiciones de servicio.

Teniendo en cuenta que la carga, las dimensiones de los muros, los materiales utilizados son idénticos en ambos sistemas, podemos referirnos para éste, a las conclusiones y recomendaciones emitidas en el apartado para el sistema modular en estribo.

\section{SISTEMA DE ARMADURAS MODULARES CON CERCOS. RECOMENDACIONES DERIVADAS DE LOS ENSAYOS REALIZADOS EN LA UNIVERSIDAD DE LIEJA}

El sistema de armaduras modulares en estribo, fabricado en Bélgica con la denominación comercial MURFOR $\mathrm{RE}$, consiste en una combinación de armaduras verticales y horizontales, se trata de una armadura prefabricada tridimensional. Está compuesta por barras de acero en forma de cerco, soldadas ortogonalmente a distancias regulares a los aceros horizontales (4), (Fig. 3a). Estas armaduras se colocan en cada hilada de bloques con el fin de obtener un recubrimiento entre las mismas (Figs. 3b, c, d y e). La solidarización del conjunto se consigue rellenando los bloques con el microhormigón.

La armadura horizontal que sirve de guía para la colocación de estos estribos tiene como función reforzar las juntas. Se conoce bien la técnica de funcionamiento de esta armadura.

El cierre de estos cercos se realiza por soldadura. Esta soldadura debe realizarse en uno de los ramales transversales, es decir, en la zona menos solicitada del estribo.

Un programa de ensayos sobre muros sometidos a flexión transversal, realizado en la Universidad de Lieja, permitió comparar el comportamiento de las armaduras modulares en estribo (tridimensionales) con el de las armaduras tradicionales. En cada uno de los casos, se 


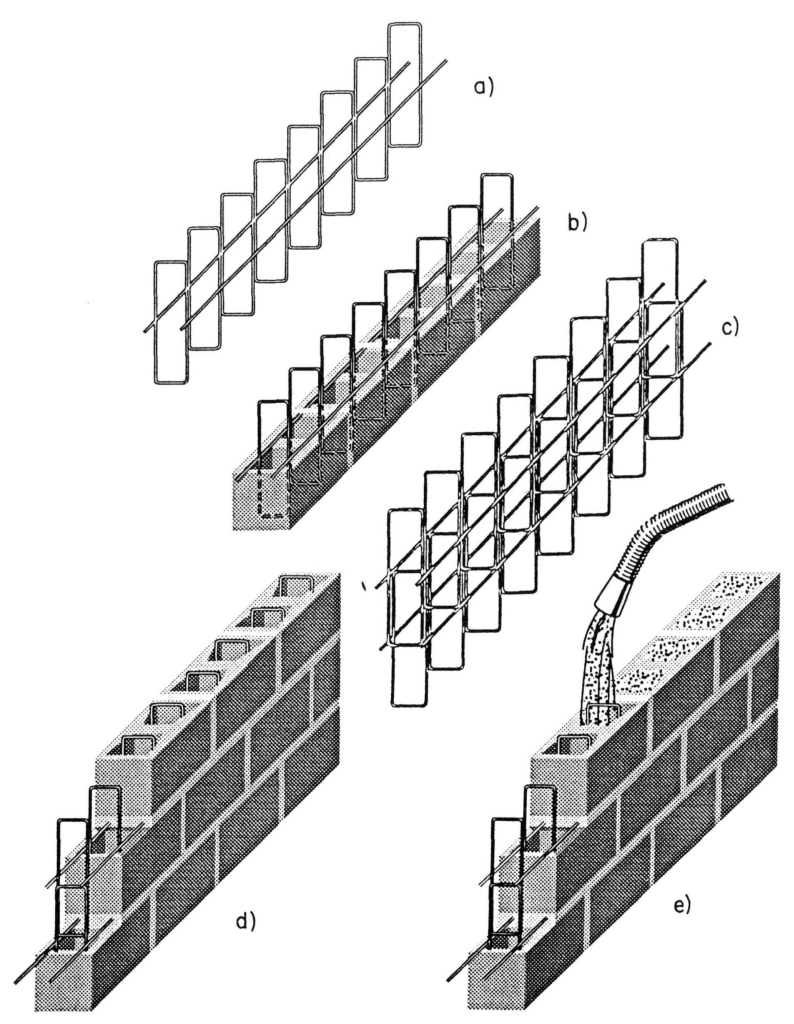

Fig. 3.-Sistema de armaduras modulares con cercos.

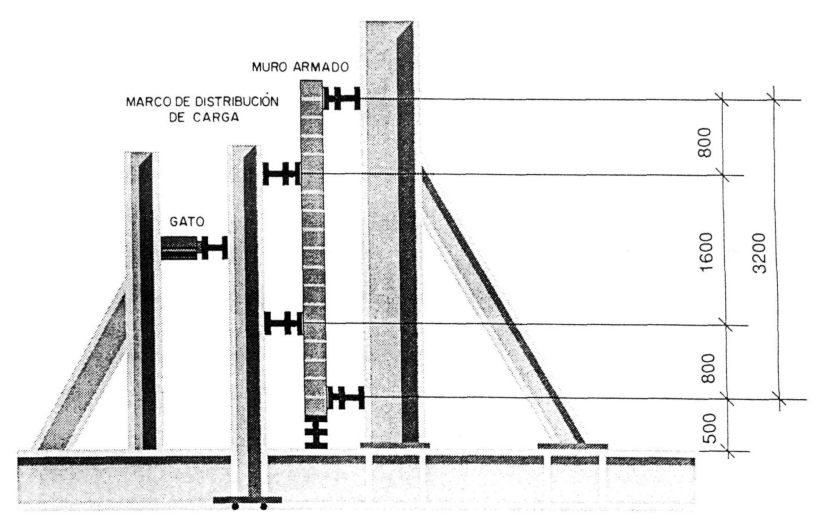

Fig. 4.-Dispositivo de carga para los ensayos sobre muros armados sometidos a flexión. eligieron los diámetros de las barras de forma que los momentos resistentes sean sensiblemente los mismos en ambos casos. Para el sistema modular en estribo, sólo se ha tenido en cuenta, en este cálculo aproximado, la contribución de la armadura más solicitada.

El dispositivo experimental utilizado se representa en la figura 4. Se trata de muros de 3,20 m de altura, cargados en la cuarta parte de la luz. Si se exceptúa la influencia del peso propio, muy reducida, se puede considerar que se trata de muros exclusivamente a flexión. Esta solicitación es bastante desfavorable para el muro; además, permite una comparación fácil entre los distintos elementos y simplifica el montaje experimental en laboratorio.

En las figuras $5 \mathrm{a}$ a $5 \mathrm{~d}$ se presenta cierto número de resultados experimentales, en forma de curvas cargasflechas. Los parámetros probados eran los siguientes:

- el sistema de armaduras;

- el diámetro de las armaduras;

- la composición del microhormigón.

Estos ensayos comparativos permitieron poner de relieve los puntos siguientes:

- El sistema de armaduras modulares con cercos (tridimensionales) se comporta tan bien como el sistema tradicional, o mejor en lo que concierne a la resistencia a la rotura.

- En general, este sistema conduce a un comportamiento más rígido que el sistema tradicional.

- La calidad del microhormigón influye a la vez en la rigidez del muro y en el momento de fisuración.

Mejorando la granulometría del microhormigón, se ha obtenido un aumento del momento de fisuración del $17 \%$ con el sistema modular con cercos.

Utilizando un aditivo a base de estireno-butadieno, se ha obtenido un aumento del $33 \%$ del momento de agrietamiento con el sistema modular en estribo; este aumento sólo es del $17 \%$ con el sistema tradicional.

A partir de estos resultados de ensayo, se pueden presentar las siguientes recomendaciones. 


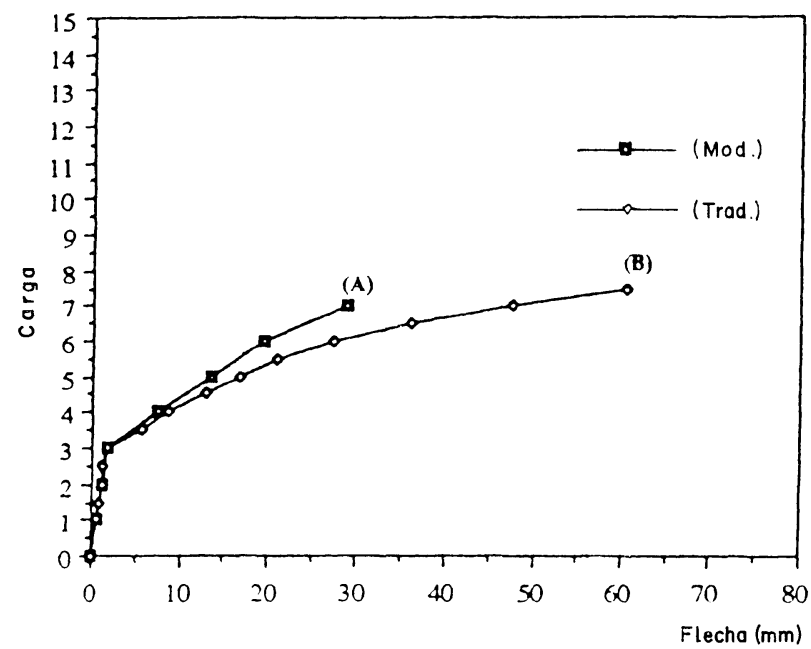

Fig. 5a.-Comparación entre las armaduras modulares con cercos de diámetro $6 \mathrm{~mm}$ y tradicionales de diámetro $8 \mathrm{~mm}$, con la mezcla $\mathrm{GN} 0,85$.

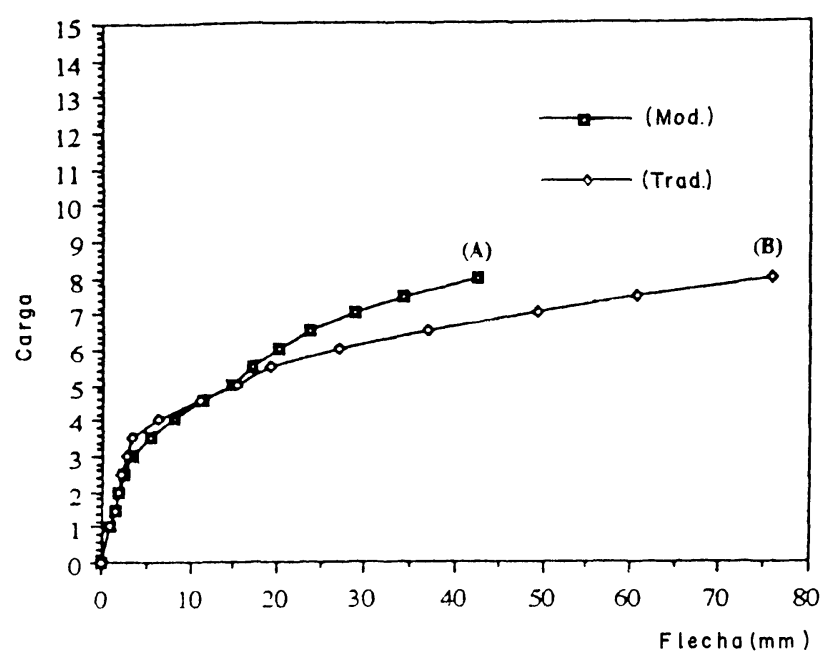

Fig. 5c.-Comparación entre las armaduras modulares con cercos de diámetro $6 \mathrm{~mm}$ y tradicionales de diámetro $8 \mathrm{~mm}$, con la mezcla GNSK 0,60.

Estas recomendaciones se refieren a los muros flexionados. Se puede considerar sin embargo que son también válidas para muros flexionados y ligeramente comprimidos.

Se pueden aplicar a muros construidos con bloques estructurales de hormigón de $19 \times 19 \times 39 \mathrm{~cm}$, cuya resistencia a la compresión es de aproximadamente $20 \mathrm{MPa}$.

Estos bloques se rellenan con un microhormigón cuyas características responden a las especificaciones de los apartados anteriores.

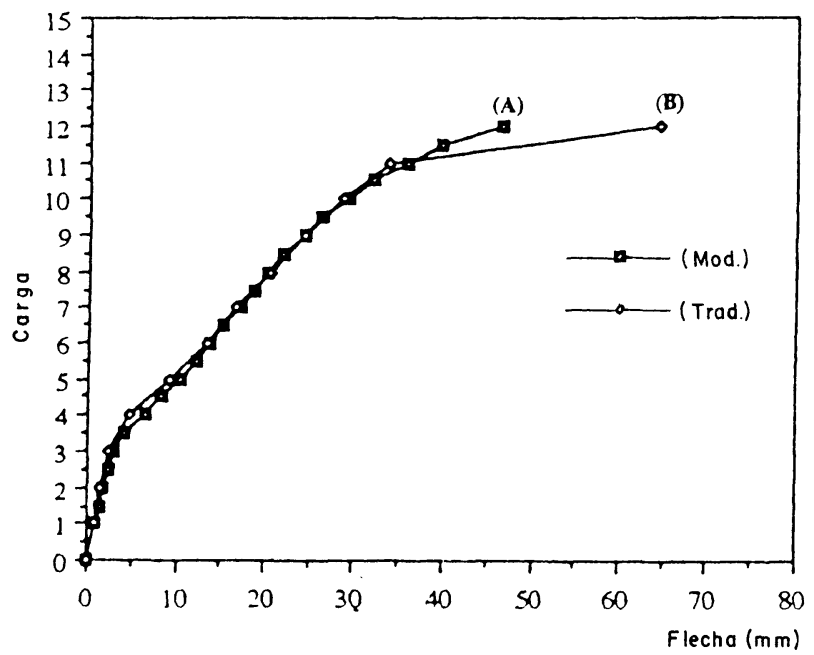

Fig. 5b.-Comparación entre las armaduras modulares con cercos de diámetro $8 \mathrm{~mm}$ y tradicionales de diámetro $10 \mathrm{~mm}$ con la mezcla GN 0,85.

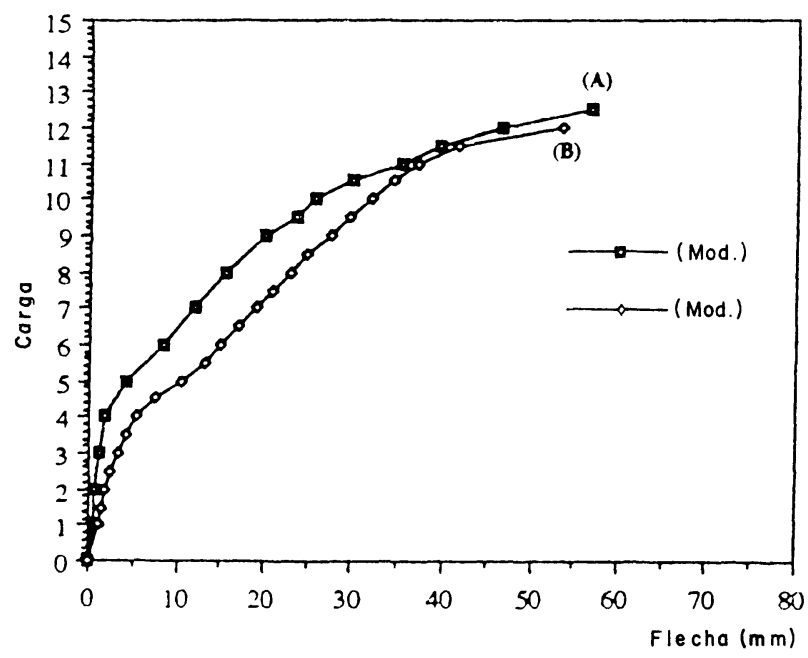

Fig. 5d.-Comparación entre las armaduras modulares con cercos de diámetro $8 \mathrm{~mm}$ y tradicionales de diámetro $10 \mathrm{~mm}$, con la mezcla GNSK 0,60.

Las armaduras tienen $6 \mathrm{u} 8 \mathrm{~mm}$ de diámetro $(8$ ó $10 \mathrm{~mm}$ en el sistema tradicional). Los ensayos mostraron que, para estas dimensiones, la longitud de recubrimiento, de aproximadamente $15 \mathrm{~cm}$, aun siendo un poco menos que el espesor de un bloque, resulta suficiente.

Los ensayos se realizaron con aceros BE 500 corrugados.

Estas recomendaciones no se aplican a los sistemas de construcción para los que no hay rejuntado de mortero entre los bloques. 

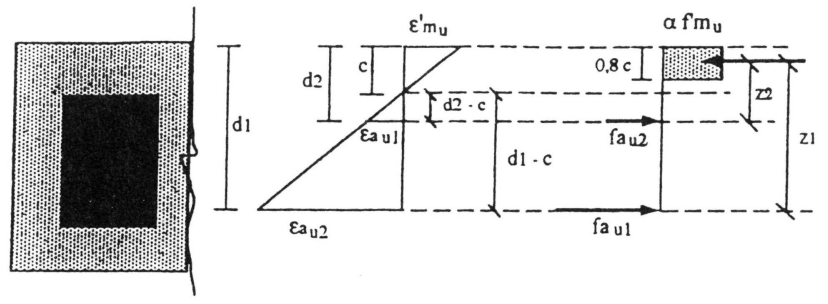

Fig. 6.-Distribución de las tensiones y de las deformaciones en un muro armado flexionado. Cálculo en estado - límite último. Sistema modular con cercos.

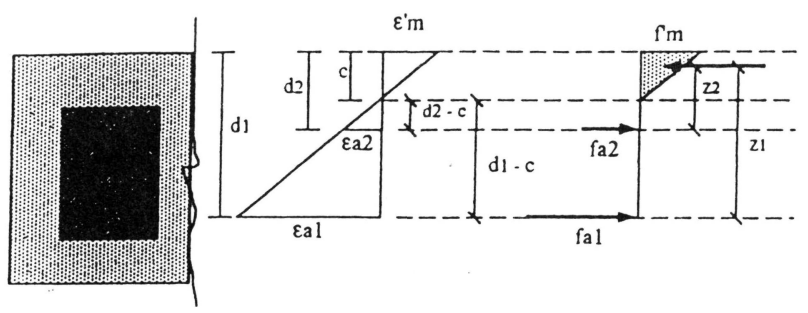

Fig. 7.-Distribución de las tensiones y de las deformaciones en un muro armado flexionado. Método de las tensiones admisibles. Sistema modular con cercos.

\section{TABLA I}

\begin{tabular}{|l|c|c|c|c|}
\hline \multicolumn{5}{|c|}{ Coeficientes parciales de seguridad para } \\
los materiales (1) \\
\hline Propiedades & Símb. & $\begin{array}{c}\text { Estado-lím. } \\
\text { último }\end{array}$ & $\begin{array}{c}\text { Cargas } \\
\text { accid. }\end{array}$ & $\begin{array}{c}\text { Deformaciones y } \\
\text { tensiones esti- } \\
\text { madas o aberturas } \\
\text { de fisuras }\end{array}$ \\
\hline $\begin{array}{l}\text { Compresión axil } \\
\text { más flexión }\end{array}$ & $\gamma \mathrm{m}$ & $\begin{array}{c}2,0^{*} \circ \\
2,3^{* *}\end{array}$ & $\begin{array}{c}1,0^{*} \text { o } \\
1,15^{* *}\end{array}$ & 1,5 \\
\hline $\begin{array}{l}\text { Resistencia al } \\
\text { cizallamiento de } \\
\text { la fábrica }\end{array}$ & $\gamma \mathrm{mv}$ & 2,0 & 1,0 & \\
\hline $\begin{array}{l}\text { Resistencia a la } \\
\text { adherencia entre } \\
\text { el microhormigón } \\
\text { y el acero }\end{array}$ & $\gamma \mathrm{mb}$ & 1,5 & 1,0 & \\
\hline $\begin{array}{l}\text { Resistencia } \\
\text { del acero }\end{array}$ & $\gamma \mathrm{ms}$ & 1,15 & 1,0 & 1,0 \\
\hline
\end{tabular}

* control especial

** control normal
El cálculo del momento máximo admisible retomado por los muros se realizó basándose en los métodos o criterios siguientes:

- cálculo al estado-límite último;

- método de las tensiones admisibles;

- criterios de servicio.

En fábrica armada, el cálculo al estado-límite último no se suele utilizar, ya que conduce a una abertura de las grietas frecuentemente demasiado importante. El principio del cálculo al estado-límite último se da en al figura 6. Es totalmente similar al adoptado en hormigón armado. La armadura intermedia trabaja, por lo general, en un cálculo en rotura. Teniendo en cuenta que se trata de bloques de hormigón, se puede adoptar $\alpha=0,85$. Los coeficientes que se van a utilizar se dan en la tabla I; se trata de los coeficientes propuestos en el proyecto de Euro-Code 6 (1).

El principio del cálculo en las tensiones admisibles se da en la figura 7. Es totalmente similar al adoptado en hormigón armado. La armadura intermedia puede estar, según los casos, ligeramente traccionada o comprimida.

Hay que tener en cuenta también las condiciones de servicio, es decir, esencialmente en nuestro caso la fisuración y las flechas transversales.

Los resultados obtenidos para los nueve muros probados se resumen en las tablas II a V.

La tabla II presenta los resultados del cálculo en estadolímite último. Se da en primer lugar la carga y el momento último obtenidos experimentalmente. Se calcula entonces el momento resistente teórico a partir de valores medios medidos de las resistencias de materiales. Se comprueba que existe buena concordancia entre resultados teóricos y experimentales y que todos los resultados teóricos son asegurables.

Se evalua seguidamente el momento resistente de cálculo a partir de los valores característicos de las resistencias y de los coeficientes $\gamma_{\mathrm{m}}$. Se deduce de ello el momento máximo admisible utilizando los coeficientes $\gamma_{\mathrm{s}}$. Se han examinado dos casos: una carga variable (el viento) para la cual $\gamma_{\mathrm{sv}}=1,5 \mathrm{y}$ una carga permanente (el empuje de tierras) para el cual $\gamma_{\mathrm{sp}}=1,35$. En ambos casos, se ha calculado la relación entre el momento último experimental y el momento máximo admisible. 
TABLA II

\begin{tabular}{|c|c|c|c|c|c|c|c|c|c|}
\hline \multicolumn{10}{|c|}{ Cálculo del momento resistente por el método de rotura } \\
\hline \multirow{2}{*}{$\begin{array}{l}M \\
n^{0}\end{array}$} & \multirow{2}{*}{ Caracteristicas } & \multicolumn{2}{|c|}{ Resultados ensayo } & \multirow{2}{*}{$\begin{array}{l}\text { Mto. resist. } \\
\text { teórico } \\
\text { Mth (KN.cm) }\end{array}$} & \multirow{2}{*}{$\begin{array}{l}\text { Mto. resist. } \\
\text { de calc. } \\
\operatorname{Mr}(\mathrm{KN} . \mathrm{cm})\end{array}$} & \multicolumn{2}{|c|}{ Aplic. 1 - Carga Variable } & \multicolumn{2}{|c|}{$\begin{array}{c}\text { Aplicac. } 2 \\
\text { Carga perman. (empuje t) }\end{array}$} \\
\hline & & $\begin{array}{l}\text { Pr exp. } \\
(\mathrm{KN})\end{array}$ & $\begin{array}{l}\text { Mr exp. } \\
(\mathrm{KN} . \mathrm{cm})\end{array}$ & & & $\begin{array}{c}\mathrm{Madm}=\mathrm{M} / 1,50 \\
(\mathrm{KN} . \mathrm{cm})\end{array}$ & $\begin{array}{l}\text { Mr exp. } \\
\text { Mv adm. }\end{array}$ & $\begin{array}{c}\text { Madm }=M / 1,35 \\
(\mathrm{KN} . \mathrm{cm})\end{array}$ & $\begin{array}{l}\text { Mr exp. } \\
\text { Mp adm }\end{array}$ \\
\hline 1 & $\begin{array}{c}\text { Bek } d=8 \mathrm{~mm} \\
\text { G1 } 150,75\end{array}$ & 127,7 & $5.108,0$ & $4.728,0$ & 2.935 & 1.957 & 2,61 & 2.174 & 2,35 \\
\hline 2 & $\begin{array}{c}\text { Trad. } \mathrm{d}=10 \mathrm{~mm} \\
\text { GN } 0,85\end{array}$ & $125,5^{*}$ & $5.020,0$ & $3.874,0$ & 2.617 & 1.745 & 2,88 & 1.939 & 2,59 \\
\hline 3 & $\begin{array}{c}\text { Trad. } d=8 \mathrm{~mm} \\
\text { GN } 0,85\end{array}$ & $75,0^{*}$ & $3.000,0$ & $2.197,0$ & 1.805 & 1.203 & 2,49 & 1.337 & 2,24 \\
\hline 4 & $\begin{array}{c}\text { Bek } \mathrm{d}=6 \mathrm{~mm} \\
\text { GN } 0,85\end{array}$ & 79,7 & $3.188,0$ & $2.934,0$ & 1.919 & 1.279 & 2,49 & 1.421 & 2,24 \\
\hline 5 & $\begin{array}{l}\text { Bek } d=8 \mathrm{~mm} \\
\text { GN } 0,85\end{array}$ & 138,0 & $5.520,0$ & $4.642,0$ & 2.800 & 1.867 & 2,96 & 2.074 & 2,66 \\
\hline 6 & $\begin{array}{c}\text { Trad. } d=8 \mathrm{~mm} \\
\text { GNSK } 0,60\end{array}$ & $80,0^{*}$ & $3.200,0$ & $2.268,0$ & 1.930 & 1.287 & 2,49 & 1.430 & 2,24 \\
\hline 7 & $\begin{array}{c}\text { Trad. } d=10 \mathrm{~mm} \\
\text { GNSK } 0,60\end{array}$ & $120,0^{*}$ & $4.800,0$ & $4.134,0$ & 2.933 & 1.955 & 2,45 & 2.173 & 2,21 \\
\hline 8 & $\begin{array}{c}\text { Bek } d=6 \mathrm{~mm} \\
\text { GNSK } 0,60\end{array}$ & $80,0^{*}$ & $3.200,0$ & $3.067,0$ & 2.079 & 1.386 & 2,31 & 1.540 & 2,08 \\
\hline 9 & $\begin{array}{c}\text { Bek } d=8 \mathrm{~mm} \\
\text { GNSK } 0,60\end{array}$ & 128,0 & $5.120,0$ & $5.026,0$ & 3.529 & 2.353 & 2,18 & 2.614 & 1,96 \\
\hline
\end{tabular}

Nota: ${ }^{*} \mathrm{El}$ ensayo se interrumpió a este nivel de carga, a causa de una flecha o de una abertura de fisuras considerada demasiado importante. Madm = momento máximo admisible.

TABLA III

\begin{tabular}{|c|c|c|c|c|c|c|}
\hline \multicolumn{7}{|c|}{ Cálculo del momento resistente por el método de rotura. Influencia de la barra intermedia } \\
\hline $\begin{array}{l}\text { Muro } \\
\text { N. }\end{array}$ & Características & $\begin{array}{l}\text { Resultados ensayo } \\
\text { Pr exp. (KN) }\end{array}$ & $\begin{array}{l}\text { Resultados ensayo } \\
\text { Mr exp. (KN.cm) }\end{array}$ & $\begin{array}{c}\text { Momento rte. } \\
\text { cálculo (KN.cm) } \\
\text { una barra M }\end{array}$ & $\begin{array}{l}\text { Momento rte. } \\
\text { cálculo (KN.cm) } \\
\text { dos barras M }\end{array}$ & $\begin{array}{l}\mathrm{M} \text { (dos barras) } \\
\mathrm{M} \text { (una barra) }\end{array}$ \\
\hline 1 & $\begin{array}{c}\text { Bek } d=8 \mathrm{~mm} \\
\text { G1 } 150,75\end{array}$ & 127,7 & $5.108,0$ & 2.563 & 2.935 & 1,15 \\
\hline 2 & $\begin{array}{c}\text { Trad. } d=10 \mathrm{~mm} \\
\text { GN } 0,85\end{array}$ & $125,5^{*}$ & $5.020,0$ & 2.617 & $\mathrm{xxxx}$ & $\mathrm{xxxx}$ \\
\hline 3 & $\begin{array}{c}\text { Trad. } d=8 \mathrm{~mm} \\
\text { GN } 0,85\end{array}$ & $75,0^{*}$ & $3.000,0$ & 1.805 & $\mathrm{xxxx}$ & $\mathrm{xxxx}$ \\
\hline 4 & $\begin{array}{c}\text { Bek } d=6 \mathrm{~mm} \\
\text { GN } 0,85\end{array}$ & 79,7 & $3.188,0$ & 1.433 & 1.919 & 1,34 \\
\hline 5 & $\begin{array}{l}\text { Bek } d=8 \mathrm{~mm} \\
\text { GN } 0,85\end{array}$ & 138,0 & $5.520,0$ & 2.530 & 2.800 & 1,11 \\
\hline 6 & $\begin{array}{c}\text { Trad. } d=8 \mathrm{~mm} \\
\text { GNSK } 0,60\end{array}$ & $80,0^{*}$ & $3.200,0$ & 1.930 & $x x x x$ & $x x x x$ \\
\hline 7 & $\begin{array}{c}\text { Trad. } \mathrm{d}=10 \mathrm{~mm} \\
\text { GNSK } 0,60\end{array}$ & $120,0^{*}$ & $4.800,0$ & 2.933 & $x x x x$ & $x x x x$ \\
\hline 8 & $\begin{array}{l}\text { Bek } d=6 \mathrm{~mm} \\
\text { GNSK } 0,60\end{array}$ & $80,0^{*}$ & $3.200,0$ & 1.474 & 2.079 & 1,41 \\
\hline 9 & $\begin{array}{l}\text { Bek } d=8 \mathrm{~mm} \\
\text { GNSK } 0,60\end{array}$ & 128,0 & $5.120,0$ & 2.680 & 3.529 & 1,32 \\
\hline
\end{tabular}

Nota: *El ensayo se interrumpió a este nivel de carga, a causa de una flecha o de una abertura de fisuras considerada demasiado importante. 
TABLA IV

\begin{tabular}{|c|c|c|c|c|c|}
\hline \multicolumn{6}{|c|}{ Cálculo del momento resistente por el método de tensiones admisibles } \\
\hline $\begin{array}{l}\text { Muro } \\
\text { N. }\end{array}$ & Características & $\begin{array}{l}\text { Resultados ensayo } \\
\text { Pr exp. (KN) }\end{array}$ & $\begin{array}{l}\text { Resultados ensayo } \\
\text { Mr exp. (KN.cm) }\end{array}$ & $\begin{array}{c}\text { Me adm. } \\
\text { fa } \lim =300 \mathrm{MPa} \\
(\mathrm{KN} . \mathrm{cm})\end{array}$ & $\begin{array}{l}\text { Mr exp. } \\
\text { Me adm. }\end{array}$ \\
\hline 1 & $\begin{array}{c}\text { Bek d=8 mm } \\
\text { G1 } 150,75\end{array}$ & 127,7 & $5.108,0$ & 1.843 & 2,8 \\
\hline 2 & $\begin{array}{c}\text { Trad. } \mathrm{d}=10 \mathrm{~mm} \\
\text { GN } 0,85\end{array}$ & $125,5^{*}$ & $5.020,0$ & 1.720 & 2,9 \\
\hline 3 & $\begin{array}{c}\text { Trad. } d=8 \mathrm{~mm} \\
\text { GN } 0,85\end{array}$ & $75,0^{*}$ & $3.000,0$ & 1.213 & 2,5 \\
\hline 4 & $\begin{array}{c}\text { Bek } d=6 \mathrm{~mm} \\
\text { GN } 0,85\end{array}$ & 79,7 & $3.188,0$ & 1.101 & 2,9 \\
\hline 5 & $\begin{array}{c}\text { Bek } d=8 \mathrm{~mm} \\
\text { GN } 0,85\end{array}$ & 138,0 & $5.520,0$ & 1.798 & 3,1 \\
\hline 6 & $\begin{array}{c}\text { Trad. } d=8 \mathrm{~mm} \\
\text { GNSK } 0,60\end{array}$ & $80,0^{*}$ & $3.200,0$ & 1.319 & 2,4 \\
\hline 7 & $\begin{array}{c}\text { Trad. } \mathrm{d}=10 \mathrm{~mm} \\
\text { GNSK } 0,60\end{array}$ & $120,0^{*}$ & $4.800,0$ & 1.981 & 2,4 \\
\hline 8 & $\begin{array}{c}\text { Bek } d=6 \mathrm{~mm} \\
\text { GNSK } 0,60\end{array}$ & 965,0 & $3.200,0$ & 1.189 & 2,7 \\
\hline 9 & $\begin{array}{c}\text { Bek } d=8 \mathrm{~mm} \\
\text { GNSK } 0,60\end{array}$ & 128,0 & $5.120,0$ & 2.019 & 2,5 \\
\hline
\end{tabular}

Nota: *El ensayo se interrumpió a este nivel de carga, a causa de una flecha o de una abertura de fisuras considerada demasiado importante. Me adm = momento máximo admisible.

TABLA V

\begin{tabular}{|c|c|c|c|c|c|c|c|}
\hline \multicolumn{7}{|c|}{ Cálculo del momento admisible en estado-límite de servicio } \\
\hline $\begin{array}{c}\text { Muro } \\
\text { N. }\end{array}$ & Características & $\begin{array}{c}\text { Resultados ensayo } \\
\text { Pr exp. (KN) }\end{array}$ & $\begin{array}{c}\text { Resultados ensayo } \\
\text { Mr exp. (KN.cm) }\end{array}$ & $\begin{array}{c}\text { Me adm. } \\
\text { fa lim=240MPa } \\
\text { FP (KN.cm) }\end{array}$ & $\begin{array}{c}\text { Mr exp. } \\
\text { Me adm. } \\
\text { FP }\end{array}$ & $\begin{array}{c}\text { Me adm. } \\
\text { fa lim=176MPa } \\
\text { FTP (KN.cm) }\end{array}$ & $\begin{array}{c}\text { Mr exp. } \\
\text { Me adm. } \\
\text { FTP }\end{array}$ \\
\hline 1 & $\begin{array}{c}\text { Bek d=8 mm } \\
\text { Gl 150,75 }\end{array}$ & 127,7 & $5.108,0$ & 1.535 & 3,3 & 1.176 & 4,3 \\
\hline 2 & $\begin{array}{c}\text { Trad. d=10 mm } \\
\text { GN 0,85 }\end{array}$ & $125,5^{*}$ & $5.020,0$ & 1.457 & 3,4 & 1.133 & 4,4 \\
\hline 3 & $\begin{array}{c}\text { Trad. d=8 mm } \\
\text { GN 0,85 }\end{array}$ & $75,0^{*}$ & $3.000,0$ & 1.002 & 3,0 & 761 & 3,9 \\
\hline 4 & $\begin{array}{c}\text { Bek d=6 mm } \\
\text { GN 0,85 }\end{array}$ & 79,7 & $3.188,0$ & 906 & 3,5 & 683 & 4,7 \\
\hline 5 & $\begin{array}{c}\text { Bek d=8 mm } \\
\text { GN 0,85 }\end{array}$ & 138,0 & $5.520,0$ & 1.501 & 3,7 & 1.150 & 4,8 \\
\hline 6 & $\begin{array}{c}\text { Trad. d=8 mm } \\
\text { GNSK 0,60 }\end{array}$ & $80,0^{*}$ & $3.200,0$ & 1.071 & 3,0 & 794 & 4,0 \\
\hline 7 & $\begin{array}{c}\text { Trad. d=10 mm } \\
\text { GNSK 0,60 }\end{array}$ & $120,0^{*}$ & $4.800,0$ & 1.622 & 3,0 & 1.222 & 3,9 \\
\hline 8 & $\begin{array}{c}\text { Bek d=6 mm } \\
\text { GNSK 0,60 }\end{array}$ & $80^{*}$ & $3.200,0$ & 965 & 3,3 & 718 & 4,5 \\
\hline 9 & $\begin{array}{c}\text { Bek d=8 mm } \\
\text { GNSK 0,60 }\end{array}$ & 128,0 & $5.120,0$ & 1.655 & 3,1 & 1.242 & 4,1 \\
\hline
\end{tabular}

Nota: *El ensayo se interrumpió a este nivel de carga, a causa de una flecha o de una abertura de fisuras considerada demasiado importante. FP $=$ fisuración perjudicial $\mathrm{FTP}=$ fisuración muy perjudicial $\mathrm{Me}$ adm $=$ momento máximo admisible 
1

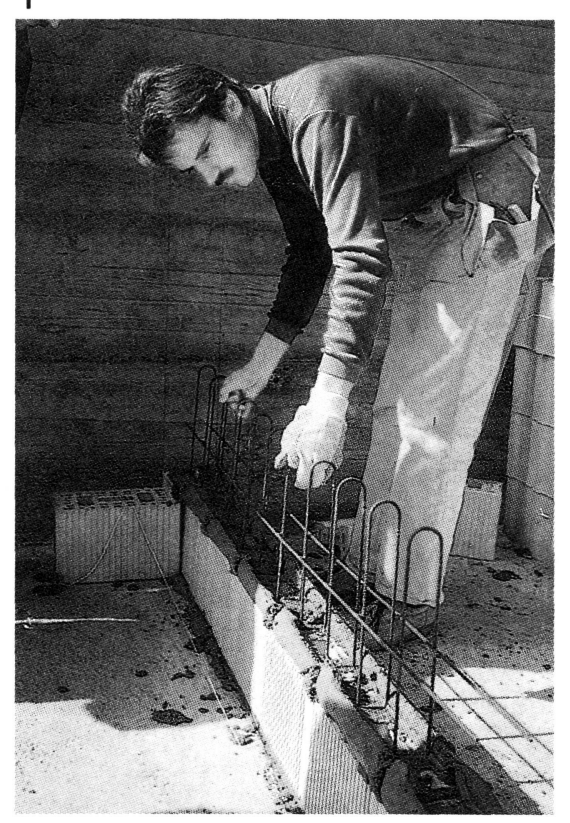

2

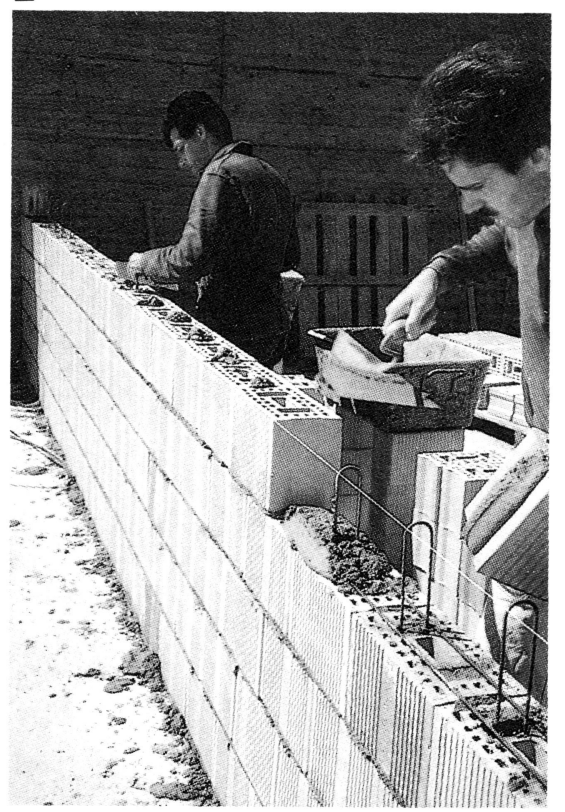

Fotos 1 y 2.-Colocación de la armadura prefabricada tridimensional Murfor RE en una fábrica de bloque cerámico.

La tabla III permite ver la influencia de la barra intermedia en la evaluación del momento resistente de cálculo. En estado-límite último, esta barra está trabajando. Se observa que, según los casos, esta influencia varía entre 11 y $41 \%$ y que, por consiguiente, no es en modo alguno despreciable.

La tabla IV presenta los resultados del cálculo por los métodos de las tensiones admisibles. Se observa que la relación $\mathrm{M}_{\mathrm{r}}$ exp/ $\mathrm{M}_{\mathrm{adm}}$ es muy próxima a la obtenida en la tabla II para el caso de la carga variable, lo cual es totalmente lógico.

En lo que concierne a las condiciones de servicio considerar las flechas transversales y la fisuración.

En cuanto a las flechas, se hace referencia a la norma inglesa BS 5628 (5), que define los valores límites L/500 ó $20 \mathrm{~mm}$, con el fin de evitar degradaciones en el acabado (revestimiento).

En lo que concierne a la fisuración, se hace referencia al reglamento francés BAEL 80 (6) utilizando los mismos criterios que para el hormigón. Se limita la tensión en los aceros en las dos situaciones siguientes:

- fisuración perjudicial $\left(\mathrm{f}_{\mathrm{a}}=240 \mathrm{MPa}\right)$;

- fisuración muy perjudicial $\left(f_{a}=176 \mathrm{MPa}\right)$.

La tabla $\mathrm{V}$ presenta los resultados obtenidos para estos dos casos límites.
Para todas estas condiciones de servicio, la condición determinante es el caso de fisuración muy perjudicial.

Por consiguiente, teniendo en cuenta el pequeño número de resultados experimentales de los que se dispone, y la fisuración en los problemas de estética y de estanquidad de las fábricas, se recomienda realizar el cálculo del muro según el método de las tensiones admisibles limitando la tensión en los aceros a $f_{a}=176 \mathrm{MPa}$. Se añaden dos imágenes de la colocación de la armadura prefabricada tridimensional Murfor RE en una fábrica de bloque cerámico (Fotos 1 y 2).

\section{BIBLIOGRAFÍA}

(1) COMMISSION OF EUROPEAN COMMUNITIES, "Eurocode 6 - Commom unified rules for reinforced masonry structures", october 1989.

(2) AITCIN, P.C., JOLICOEUR, G. et MERCIER, M. "Tecnología de granulados", Les Editions du Griffon d'argile Inc., Québec, Canadá, 1983, 372 P.

(3) AMRHEIN J.E., "Reinforced masonry engineering handbook", Masonry Institue of America, Los Angeles, USA, 1983, 461 p.

(4) REINLE, E. et LOOCK, R.C., "Reinforced apparatus for a masonry wall”, United States Patent 4.939881 July, 1990.

(5) BRITISH STANDARDS INSTITUTION, "Code of practice for the use of mansory”, BS 5628, London, 1985.

(6) REGLAS BAEL 80, "Reglas técnicas de concepción y de cálculo de las obras y construcciones en hormigón armado según el método de los estados límites", 2. ${ }^{\circ}$ edition, París, 1982, 315 páginas. 